\title{
Research Article Distortion-Free 1-Bit PWM Coding for Digital Audio Signals
}

\author{
Andreas Floros ${ }^{1}$ and John Mourjopoulos ${ }^{2}$ \\ ${ }^{1}$ Department of Computer Science, Ionian University, Plateia Tsirigoti 7, 49100 Corfu, Greece \\ ${ }^{2}$ Audio Technology Group, Department of Electrical and Computer Engineering, University of Patras, 26500 Rio Patras, Greece
}

Received 15 June 2006; Revised 1 December 2006; Accepted 13 March 2007

Recommended by Sven Nordholm

\begin{abstract}
Although uniformly sampled pulse width modulation (UPWM) represents a very efficient digital audio coding scheme for digitalto-analog conversion and full-digital amplification, it suffers from strong harmonic distortions, as opposed to benign nonharmonic artifacts present in analog PWM (naturally sampled PWM, NPWM). Complete elimination of these distortions usually requires excessive oversampling of the source PCM audio signal, which results to impractical realizations of digital PWM systems. In this paper, a description of digital PWM distortion generation mechanism is given and a novel principle for their minimization is proposed, based on a process having some similarity to the dithering principle employed in multibit signal quantization. This conditioning signal is termed "jither" and it can be applied either in the PCM amplitude or the PWM time domain. It is shown that the proposed method achieves significant decrement of the harmonic distortions, rendering digital PWM performance equivalent to that of source PCM audio, for mild oversampling (e.g., $\times 4$ ) resulting to typical PWM clock rates of $90 \mathrm{MHz}$.
\end{abstract}

Copyright (c) 2007 A. Floros and J. Mourjopoulos. This is an open access article distributed under the Creative Commons Attribution License, which permits unrestricted use, distribution, and reproduction in any medium, provided the original work is properly cited.

\section{INTRODUCTION}

Over the last decades, the use of 1-bit audio signals has emerged as an attractive practical alternative to multibit pulse code modulation (PCM) audio, which up to now was considered as the de facto format for the representation of such data. The advantages of a pulse-stream representation for digital audio originate from the simpler hardware implementations with respect to the required audio performance. For example, analog-to-digital (ADC) and digital-to-analog (DAC) conversion systems with the increased requirements imposed in dynamic range and bandwidth can be efficiently implemented using 1-bit digital storage formats (i.e., in the form of direct stream digital-DSD [1], which is based upon sigma-delta modulation-SDM [2]).

Similarly, conversion of audio to 1-bit pulse width modulation (PWM) streams introduces comparable practical implementation advantages for the realization of DACs [3] and other components in the audio chain, especially alldigital amplifiers, since the PWM pulse-stream can be directly amplified using power switch transistors [4]. Theoretically, any switching power stage has $100 \%$ efficiency. In practice, no ideal power switch exists and such implementations result into an amount of power loss taking place when the power switches cross their linear range [5]. Hence, although SDM requires no linearization for achieving acceptable distortion levels, PWM audio coding represents a more attractive digital amplification format, since it incorporates lower number of power switch transitions. More specifically, as it will be discussed in the following section, the 1-bit PWM stream representation requires two different clocks: the sampling frequency $f_{s}$ that equals to the PWM pulse transitions repetition and a much higher clock $f_{p}$ that determines the exact time instances of these transitions. On the contrary, for SDM both the sampling and the pulse repetition rates are the same with a value in the range of $2.8 \mathrm{MHz}$. This increased pulse repetition rate imply higher power dissipation and lower power efficiency, due to the very frequent transition of the MOSFET switches implementing the final output stage over their linear operating region [6]. Furthermore, PWM coding also overcomes potential problems associated with SDM audio coding, such as out-of-band noise amplification, zero-level input signal idle tones and limit cycles responsible for audible baseband tones $[7,8]$.

Although many all-digital amplification commercial systems are now appearing, the theoretical implications of using such 1-bit data are not very well understood and usually these systems employ practical "rule of thumb" solutions to suppress unwanted side effects and distortions generated 


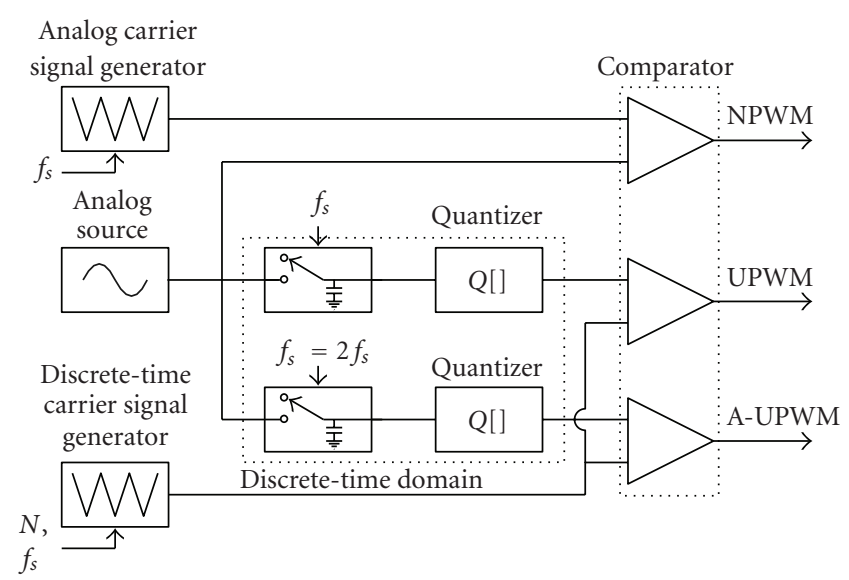

FIgURE 1: Alternative PWM modulation schemes.

from the conversion of the better understood multibit PCM format into 1-bit signal [9].

Focusing on PWM conversion, the inherently nonlinear nature of this process introduces harmonic and nonharmonic distortions [10], which render the audio performance unsuitable for most applications. Although some distortion compensating strategies have been proposed [11, 12], none of them has achieved complete elimination of PWM distortions and most implementations rely on significant increase of the modulators' switching frequency. However, this approach proportionally increases the system complexity, introduces electromagnetic interference problems, and negates the basic PWM advantage over SDM, as it decreases the overall digital amplification efficiency, due to the increment of the PWM pulse repetition frequency [13].

The work here attempts to overcome the above problems and to improve understanding of digital audio PWM. It introduces a novel analytic approach, which allows exact description of the PWM pulse stream as well as prediction and suppression of distortion artifacts of such audio signals without excessive increment of the pulse repetition frequency, starting from the following initial assumptions.

(a) The digital audio source will be in the widely employed PCM format (typically sampled at $f_{s}=44.1 \mathrm{kHz}$ and quantized using $N=16$ bit).

(b) The case of regularly sampled (discrete-time) PWM conversion will be examined (uniformly sampled PWM, UPWM), appropriate for mapping from the sampled PCM audio data.

(c) The UPWM format can be related to the inherently analog naturally sampled PWM (NPWM), which traditionally has been analyzed and employed in many communication applications [14]. Due to the asymmetric positioning of the NPWM pulse edges, the asymmetric uniformly sampled PWM (A-UPWM) must be also examined $[15,16]$, as shown in Figure 1.

(d) As it is known, NPWM generates only nonharmonic type distortions, which can be easily eliminated from the audio band by appropriately increasing the modulation switch- ing frequency [17]. However, UPWM and A-UPWM being discrete-time processes, it is also well known to generate additional harmonic distortions [10, 18]. Furthermore, assuming that the PCM audio data do not posses any form of distortions, it would be sensible to consider here conditions under which the mapping error between PCM and A-UPWM would be eliminated. Nevertheless, it is analytically shown here (see the appendix) that this condition is only satisfied for a full-scale DC signal, so that it will not be applicable to any practical audio data. Therefore, the work here will be mainly concerned with the minimization of errors between NPWM and the equivalent A-UPWM conversion. It will be shown that such an approach will also allow optimal mapping between the PCM and UPWM.

The work is organized as follows: in Section 2, a novel analytic description of the A-UPWM and NPWM coding is introduced. It is also shown (Section 3) that the A-UPWMinduced harmonic distortions are generated due to the sampling process applied during the PCM-to- A-UPWM mapping. Hence, a novel principle for minimizing such signalrelated distortions in 1-bit digital PWM signals is introduced in Section 4, having some parallels to the dithering principle employed for minimizing amplitude quantization artifacts in multibit PCM conversion [19]. This principle can be also expressed as controlled jittering of the UPWM pulse transition edges, and hence it is termed "jithering." Section 5 presents typical performance results of the proposed method, showing that it achieves acceptable levels of signal-dependent (harmonic) UPWM distortions under all practical conditions.

\section{PWM CONVERSION FUNDAMENTALS}

Legacy PWM represents data as width-modulated pulses generated by the comparison of the analog or digital audio waveform with a periodic carrier signal of fundamental frequency $f_{s}(\mathrm{~Hz})$, as is shown in Figure 1. More specifically, the switching instances of the PWM pulses are defined by the intersection of the input signal and the carrier waveform. For double-edged PWM considered here, the carrier should be of triangular shape, while depending on the analog or digital nature of the input, it should be an analog or a discrete-time signal, respectively.

Assuming a PCM input signal, bounded in the range of $\left[0, S_{\max }\right]$, sampled at $f_{s}^{\prime}=2 f_{s}$ and quantized to $N$ bit, the audio information will be represented by $2^{N}$ discrete amplitude levels. In order to preserve this information after PWM conversion, the PWM pulse stream should be also quantized in the time domain with an equivalent resolution. Thus, within each time interval $T_{s}^{\prime}=1 / f_{s}^{\prime}, 2^{N}$ different equally spaced intersection values should be allowed between the carrier and the digital input samples. Following this argument, the carrier waveform will be a discrete-time signal of sampling frequency $f_{p}=1 / T_{p}(\mathrm{~Hz})$, where

$$
T_{p}=\frac{T_{s}}{2\left(2^{N}-1\right)}=\frac{T_{s}^{\prime}}{\left(2^{N}-1\right)},
$$




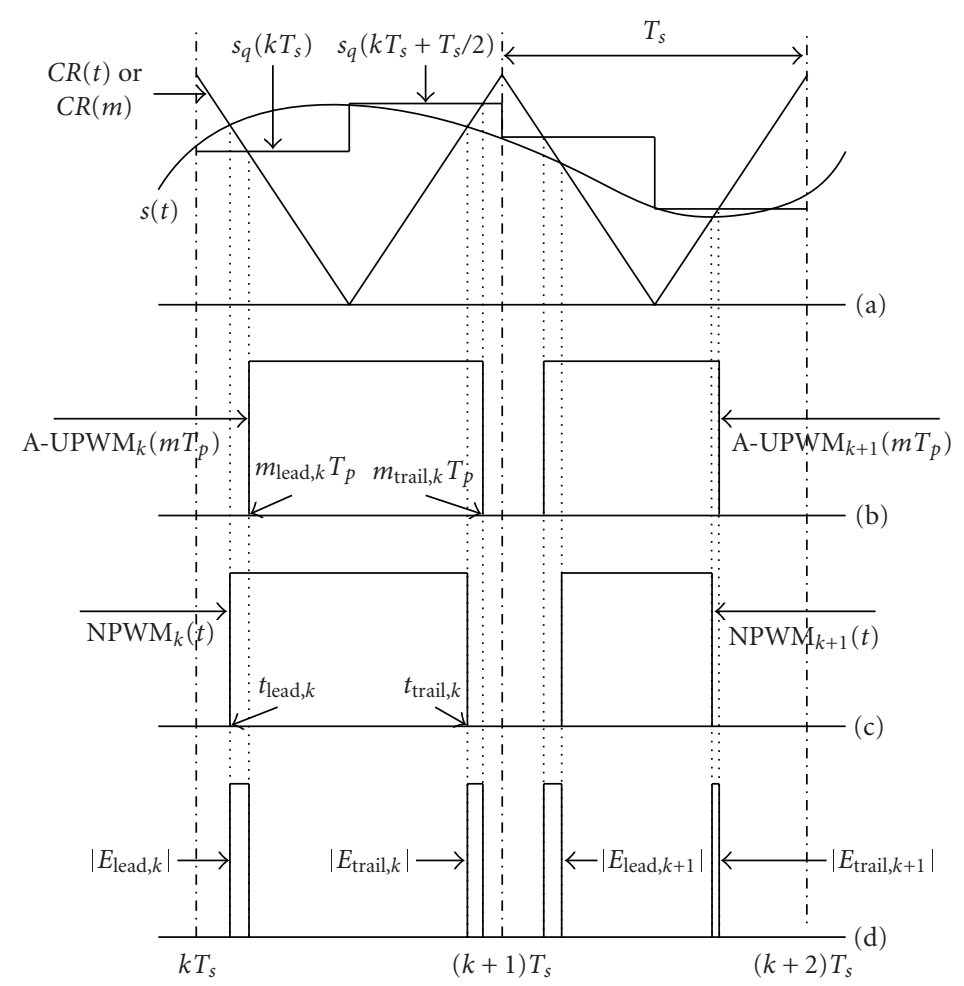

FIGURe 2: Typical audio waveforms: (a) analog/digital audio and modulation carrier (b) A-UPWM (c) NPWM (d) absolute A-UPWM to NPWM difference.

and within the $k$ th switching period $T_{s}$ it can be expressed as

$$
C R_{k}(m)=\left\{\begin{array}{l}
-S_{\max } \frac{\left[m-2 k\left(2^{N}-1\right)\right]}{2^{N}-1}+S_{\max }, \\
\quad \text { for } 2 k\left(2^{N}-1\right) \leq m \leq(2 k+1)\left(2^{N}-1\right), \\
S_{\max } \frac{\left[m-2 k\left(2^{N}-1\right)\right]}{2^{N}-1}-S_{\max }, \\
\quad \text { for }(2 k+1)\left(2^{N}-1\right) \leq m \leq 2(k+1)\left(2^{N}-1\right),
\end{array}\right.
$$

where $m$ is the PWM time-domain discrete-time integer variable defined for $[0, \infty)$.

In such a case, the leading and trailing edges of the $k$ th PWM pulse (see Figure 2) will be defined at integer multiples $m_{\text {lead, } k}$ and $m_{\text {trail, } k}$ of the period $T_{p}$ defined as

$$
\begin{aligned}
s_{q}\left(k T_{s}\right) & =C R_{k}\left(m_{\text {lead }, k}\right), \\
s_{q}\left(k T_{s}+\frac{T_{s}}{2}\right) & =C R_{k}\left(m_{\text {trail }, k}\right),
\end{aligned}
$$

where $s_{q}\left(k T_{s}\right)$ and $s_{q}\left(k T_{s}+T_{s} / 2\right)$ are the digital input samples. Using (2) and (3), the leading and trailing edge instances of the $k$ th PWM pulse will be

$$
\begin{aligned}
m_{\text {lead }, k} T_{p} & =\left(2 k+1-\frac{s_{q}\left(k T_{s}\right)}{S_{\max }}\right)\left(2^{N}-1\right) T_{p} \\
& =\left(2 k+1-\frac{s_{q}\left(k T_{s}\right)}{S_{\max }}\right) \frac{T_{s}}{2} \\
m_{\text {trail }, k} T_{p} & =\left(2 k+1+\frac{s_{q}\left(k T_{s}+T_{s} / 2\right)}{S_{\max }}\right) \frac{T_{s}}{2}
\end{aligned}
$$

Assuming now an analog input signal $s(t)$, its intersection with the carrier signal can occur at any time instance within each period $T_{s}^{\prime}$, the carrier waveform of (2) being defined also as an analog signal. Following a similar analysis to the one performed for digital inputs, the two intersection instances (one in each half of the period $T_{s}$ ) between the signal $s(t)$ and the carrier $C R_{k}(t)$ will be given by the expressions

$$
\begin{aligned}
& t_{\text {lead }, k}=\frac{T_{s}}{2}\left(2 k+1-\frac{s\left(t_{\text {lead }, k}\right)}{S_{\max }}\right), \\
& t_{\text {trail }, k}=\frac{T_{s}}{2}\left(2 k+1+\frac{s\left(t_{\text {trail }, k}\right)}{S_{\max }}\right) .
\end{aligned}
$$

Due to the time irregularity of the input signal sampling process performed at the time instances $t_{\text {lead, } k}$ and $t_{\text {trail }, k}$, the above process is called naturally sampled PWM (NPWM). Each NPWM pulse within the $k$ th switching period $T_{s}$ can be expressed as

$$
\operatorname{NPWM}_{k}(t)=A\left[u\left(t-t_{\text {lead }, k}\right)-u\left(t-t_{\text {trail }, k}\right)\right],
$$


where $A$ is the amplitude of the NPWM pulses and $u(t)$ the analog-time step function defined as

$$
u(t)= \begin{cases}1, & t \geq 0, \\ 0, & \text { otherwise. }\end{cases}
$$

On the other hand, in the case of digital input signals, the regularly spaced sampling instances $k T_{s}$ and $k T_{s}+T_{s} / 2$ generate the asymmetric uniformly sampled PWM (A-UPWM) expressed as

$$
\begin{aligned}
& A-\mathrm{UPWM}_{k}(m) \\
&=A\left\{u\left[m-\left(2 k+1-a_{q}\left(k T_{s}\right)\right)\left(2^{N}-1\right)\right]\right. \\
&\left.\quad-u\left[m-\left(2 k+1+a_{q}\left(k T_{s}+\frac{T_{s}}{2}\right)\right)\left(2^{N}-1\right)\right]\right\},
\end{aligned}
$$

where $u(m)$ is the discrete-time step function and $a_{q}\left(k T_{s}\right)$ is the normalized input signal amplitude defined by the ratio $s_{q}\left(k T_{s}\right) / S_{\max }$. Assuming that the sampling frequency $f_{s}^{\prime}$ of the digital input data is equal to the carrier fundamental pe$\operatorname{riod} f_{s}$, then both the leading and trailing edges of the PWM pulses will be modulated by a single quantized input signal value $s_{q}\left(k T_{s}\right)$. This produces the well-known case of the uniformly sampled PWM (UPWM), which is described in the time domain by (8) by setting $a_{q}\left(k T_{s}+T_{s} / 2\right)=a_{q}\left(k T_{s}\right)$ [18].

\section{UPWM-INDUCED DISTORTIONS}

Let us now compare the time-domain waveforms of the NPWM and A-UPWM streams, as described by (6) and (8). Given that the amplitude of the PWM pulses in both modulation schemes is kept constant (and equal to $A$ ) within each switching interval, we can define their time-domain difference in terms of absolute time values (see Figure 2) as

$$
E_{k}=E_{\text {lead }, k}+E_{\text {trail }, k}
$$

where

$$
\begin{aligned}
& E_{\text {lead }, k}=A\left(t_{\text {lead }, k}-m_{\text {lead }, k} T_{p}\right), \\
& E_{\text {trail }, k}=A\left(t_{\text {trail }, k}-m_{\text {trail }, k} T_{p}\right) .
\end{aligned}
$$

Using the set of (4) and (5), the above expressions give

$$
\begin{aligned}
& E_{\text {lead }, k}=\frac{A T_{s}}{2 S_{\max }}\left[s_{q}\left(k T_{s}\right)-s\left(t_{\text {lead }, k}\right)\right], \\
& E_{\text {trail }, k}=\frac{A T_{s}}{2 S_{\max }}\left[s\left(t_{\text {trail }, k}\right)-s_{q}\left(k T_{s}+\frac{T_{s}}{2}\right)\right] .
\end{aligned}
$$

Given that the error $\varepsilon_{l, k}$ and $\varepsilon_{t, k}$ generated by the amplitude quantization of the discrete time values $s\left(k T_{s}\right)$ and $s\left(k T_{s}+T_{s} / 2\right)$ to the digital samples $s_{q}\left(k T_{s}\right)$ and $s_{q}\left(k T_{s}+T_{s} / 2\right)$ is expressed as [20]

$$
\begin{aligned}
& \varepsilon_{l, k}=s\left(k T_{s}\right)-s_{q}\left(k T_{s}\right), \\
& \varepsilon_{t, k}=s\left(k T_{s}+\frac{T_{s}}{2}\right)-s_{q}\left(k T_{s}+\frac{T_{s}}{2}\right),
\end{aligned}
$$

where $-\mathrm{LSB} / 2 \leq \varepsilon_{l, k} \leq \mathrm{LSB} / 2$ and $-\mathrm{LSB} / 2 \leq \varepsilon_{t, k} \leq \mathrm{LSB} / 2$, with LSB presenting the least significant bit of the input PCM data, (11) give:

$$
\begin{aligned}
& E_{\text {lead }, k}=\frac{A T_{s}}{2 S_{\max }}\left[s\left(k T_{s}\right)-s\left(t_{\text {lead }, k}\right)-\varepsilon_{l, k}\right], \\
& E_{\text {trail }, k}=\frac{A T_{s}}{2 S_{\max }}\left[s\left(t_{\text {trail }, k}\right)-s\left(k T_{s}+\frac{T_{s}}{2}\right)+\varepsilon_{t, k}\right] .
\end{aligned}
$$

By observing the above equations, it is obvious that the time domain difference between A-UPWM and NPWM in each switching period will be due to two independent but simultaneously acting mechanisms: (a) the amplitude-domain quantization of the input signal affecting the A-UPWM conversion, expressed by the quantization error terms $\varepsilon_{l, k}$ and $\varepsilon_{t, k}$, and (b) the difference of the sampling instances between the NPWM (i.e., $t_{\text {lead }, k}$ and $t_{\text {trail }, k}$ ) and A-UPWM (i.e., $k T_{s}$ and $\left.k T_{s}+T_{s} / 2\right)$.

Considering the first mechanism, it is clear that in the case of NPWM modulation, the analog (and continuous) nature of the input signal's amplitude will result to similarly continuous time variables $t_{\text {lead, } k}$ and $t_{\text {trail }, k}$, which will define the NPWM pulse transitions. On the contrary, in the case of A-UPWM, the quantized (and discontinuous) nature of the input signal amplitude will result to discrete time values $m_{\text {lead, } k} T_{p}$ and $m_{\text {trail }, k} T_{p}$ which will define the exact positions of the A-UPWM pulse edges in the time axis. Hence, given that $T_{p}$ represents the shorter A-UPWM pulse possible time duration that corresponds to the minimum amplitude value defined for PCM coding (i.e., the PCM least significant bitLSB), this interval can be termed as the least significant time transition (LST) for the A-UPWM coding.

Moreover, as can be observed from (11), the mapping of the amplitude quantization of the PCM signals $s_{q}\left(k T_{s}\right)$ and $s_{q}\left(k T_{s}+T_{s} / 2\right)$ into discrete time variables has the typical form of the well-known amplitude quantization. As it is known, the error generated by such quantization, under certain assumptions (which are generally satisfied by any digital audio signal), will produce noise that has broadband nature and with amplitude roughly equal to $6 N$ [21]. Hence when mapping $N$-bit quantized values into the discrete time domain as given by (1), under the same assumptions, the signal noise floor level will not be affected.

Considering now the second mechanism, it is clear that in the case of the NPWM, the pulse edges coincide with the time instances at which the input signal is sampled and fed to the NPWM modulator and this natural (i.e., continuous and nonregular) sampling will result to a finely sampled signal which in effect will generate only the well-known intermodulation products [10] at frequencies

$$
f=a x\left(2 f_{s}\right)-b \times f_{\text {in }},
$$

where $a, b$ are nonzero integers and $f_{\text {in }}$ is the input signal frequency. On the contrary, in the case of A-UPWM, the sampling of the discrete PCM data at regular time instances will result to an accumulated shifting of the PWM-pulse edges (with respect to the NPWM sampling), which generates a signal-dependent FM-type modulation [15], resulting to the 
rise of the well-known harmonic distortion. It should be also noted that the amplitude of the intermodulation and harmonic distortion artifacts is not affected in any way by the quantization resolution employed. Nevertheless, the reduction of the quantization resolution $N$, can render these distortion artifacts nonaudible, due to masking by the increased noise floor level [22].

\section{A-UPWM DISTORTION MINIMIZATION}

Following the analysis in the previous section, a possible AUPWM harmonic distortion suppression scheme is to approximate the A-UPWM sampling instances with those derived using the NPWM coding scheme. This approximation can be performed by minimizing the time-domain difference $E_{k}$ of A-UPWM and NPWM expressed using (9) and (10) as

$$
E_{k}=A\left[\left(t_{\text {lead }, k}-m_{\text {lead }, k} T_{p}\right)+\left(t_{\text {trail }, k}-m_{\text {trail }, k} T_{p}\right)\right]
$$

or equivalently, using the set of (11):

$E_{k}=\frac{A T_{s}}{2 S_{\max }}\left[\left(s_{q}\left(k T_{s}\right)-s\left(t_{\text {lead }, k}\right)\right)+\left(s\left(t_{\text {trail }, k}\right)-s_{q}\left(k T_{s}+\frac{T_{s}}{2}\right)\right)\right]$.

Obviously, the minimization of $E_{k}$ can be efficiently achieved when the sampling interval $T_{s}$ decreases, that is, when using sufficiently high oversampling, typically by a factor of $\times 64$ [22]. In this case, the derived oversampled signal better approximates its original analog equivalent, hence the A-UPWM stream pulse transition instances are closer to the NPWM pulse edges. However, in this case, (1) results into extremely high PWM clock rates $f_{p}$ that are impossible to be realized in practice.

Here, a novel solution is proposed, based on the following two alternative strategies: (a) in the amplitude domain, by proper modification of the amplitude of the input samples $s_{q}\left(k T_{s}\right)$ and $s_{q}\left(k T_{s}+T_{s} / 2\right)$. This process is equivalent to adding digital dither prior to A-UPWM conversion, or (b) in the time domain, by proper displacement (jittering) of the A-UPWM pulse edges.

Hence, the generic term "jither" can be employed to describe both minimization strategies [23]. Such minimization will remove all harmonic artifacts without affecting the nonharmonic distortions inherent to the "NPWM-like" nature of the "jithered" A-UPWM, which however can be easily eliminated from the audio band by simply doubling the conversion switching frequency. Thus, the proposed PWM distortion minimization method is based on the structure shown in Figure 3, having the following stages.

(i) A "jither" module, implemented in either the PCMamplitude or the PWM-time domain. This renders AUPWM equivalent to NPWM and removes all PWMinduced harmonic distortions. Especially if UPWM conversion is considered, (which is the typical case in digital audio applications) an $\times 2$ oversampling process must be also employed within this module in order to produce the A-UPWM waveform which does not affect the final PWM rate.

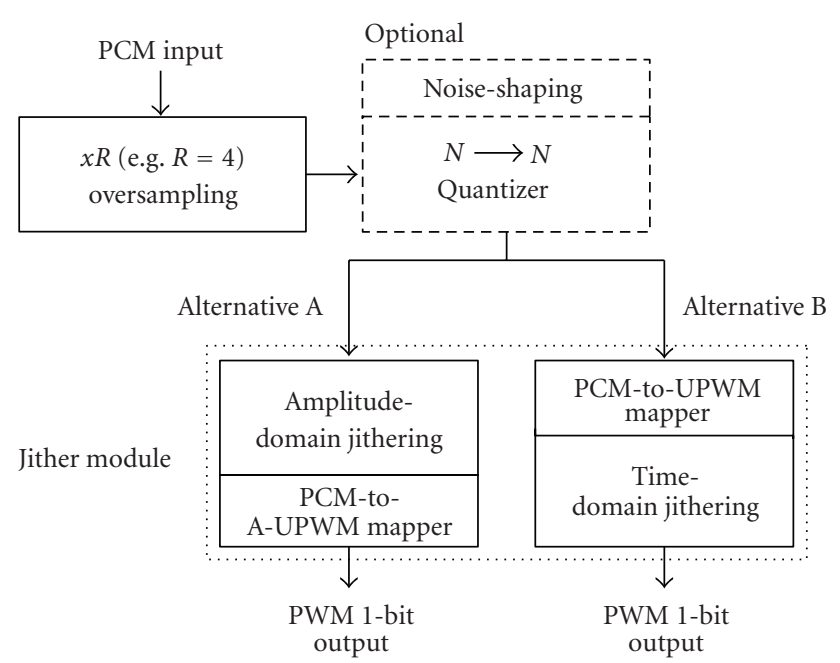

Figure 3: Block diagram of the proposed PWM correction chain.

(ii) An $\times R$ oversampling stage (typically $R=2$ ) which will shift the NPWM-like nonharmonic intermodulation artifacts outside the audio band.

(iii) An optional input PCM amplitude quantizer stage (e.g., from $N=16$ to $N^{\prime}=8$ bit), so that the final PWM clock rates can be kept to desirable low values. More specifically, according to (1), the PWM clock rate in the case of $N=16$ bit equals to $5.7 \mathrm{GHz}(11.5 \mathrm{GHz}$ when $\times 2$ oversampling is applied), which may prove to be prohibitive for practical implementations. For the reduction of these rates to feasible values, the preconditioned samples must be requantized to 8-bit prior to the PCM-to-A-UPWM mapping. However, in this case, provided that the 8-bit resolution results into audible quantization error levels and relative poor audio quality, this process must be combined with (a) oversampling in the PCM domain (prior to the "jither" module) for reducing the overall quantization error level and (b) noise-shaping techniques [24] for effectively spreading the quantization error to less obtrusive (i.e., higher frequency) areas of the audio spectrum using conventional FIR filters. As presented in [22], a 3rd order noise shaper can significantly improve the 8-bit PCM-to-PWM mapping in terms of quantization noise audibility.

In the following sections, a more detailed analysis of the "jither" module in both amplitude and time domains is given.

\section{1. "Jither" addition in the amplitude domain}

Let us assume that the input to an A-UPWM coder is a signal sampled at a rate $2 f_{s}$ with resolution $N$ bit, described by the samples $s_{q}\left(k T_{s}\right)$ and $s_{q}\left(k T_{s}+T_{s} / 2\right)$ in each $T_{s}$ interval. The minimization of the NPWM and A-UPWM difference $E_{k}$ expressed by (16) can be achieved by adding appropriately evaluated $N$-bit quantized "jither" values $g_{\text {lead }}\left(k T_{s}\right)$ and $g_{\text {trail }}\left(k T_{s}+T_{s} / 2\right)$ to the corresponding input signal samples $s_{q}\left(k T_{s}\right)$ and $s_{q}\left(k T_{s}+T_{s} / 2\right)$ prior to A-UPWM conversion, 
hence producing the "jithered" values $s_{q}^{\prime}\left(k T_{s}\right)$ and $s_{q}^{\prime}\left(k T_{s}+\right.$ $\left.T_{s} / 2\right)$ as

$$
\begin{aligned}
s_{q}^{\prime}\left(k T_{s}\right) & =s_{q}\left(k T_{s}\right)+g_{\text {lead }}\left(k T_{s}\right), \\
s_{q}^{\prime}\left(k T_{s}+\frac{T_{s}}{2}\right) & =s_{q}\left(k T_{s}+\frac{T_{s}}{2}\right)+g_{\text {trail }}\left(k T_{s}+\frac{T_{s}}{2}\right) .
\end{aligned}
$$

As previously mentioned, both $g_{\text {lead }}\left(k T_{s}\right)$ and $g_{\text {trail }}\left(k T_{s}+T_{s} / 2\right)$ values are evaluated for concurrently minimizing both terms $E_{\text {lead, } k}$ and $E_{\text {trail, } k}$ of the difference between NPWM and AUPWM. Considering constant sampling period $\left(T_{s}\right)$ values and following (11), the above minimization is expressed as

$$
\begin{gathered}
\left|s_{q}^{\prime}\left(k T_{s}\right)-s\left(t_{\text {lead }, k}\right)\right| \leq \frac{\mathrm{LSB}}{2}, \\
\left|s\left(t_{\text {trail }, k}\right)-s_{q}^{\prime}\left(k T_{s}+\frac{T_{s}}{2}\right)\right| \leq \frac{\mathrm{LSB}}{2} .
\end{gathered}
$$

It should be noted that the NPWM and A-UPWM difference minimization is theoretically limited within the range [- LSB /2, LSB /2], due to the $N$-bit quantization of the digital samples $s_{q}^{\prime}\left(k T_{s}\right)$ and $s_{q}^{\prime}\left(k T_{s}+T_{s} / 2\right)$.

\section{2. "Jither" addition in the PWM time domain}

Alternatively, the NPWM and A-UPWM difference minimization expressed by (15) can be performed directly in the PWM domain by "jittering" the leading and trailing edge of the $k$ th A-UPWM pulse by the quantities $J_{\text {lead, } k} T_{p}$ and $J_{\text {trail }, k} T_{p}(\mathrm{sec})$, where $J_{\text {lead, } k}$ and $J_{\text {trail }, k}$ are integer indices expressing the time displacement of the PWM pulse edges as multiples of the LST. In such a case, it is required that these indices are calculated using the expressions

$$
\begin{aligned}
& \left|t_{\text {lead }, k}-m_{\text {lead }, k}^{\prime} T_{p}\right| \leq \frac{\mathrm{LST}}{2} \\
& \left|t_{\text {trail }, k}-m_{\text {trail }, k}^{\prime} T_{p}\right| \leq \frac{\mathrm{LST}}{2}
\end{aligned}
$$

where the integer indices

$$
\begin{aligned}
& m_{\text {lead }, k}^{\prime}=m_{\text {lead }, k}-J_{\text {lead }, k}, \\
& m_{\text {trail }, k}^{\prime}=m_{\text {trail }, k}+J_{\text {trail }, k},
\end{aligned}
$$

define the "jittered" positions of the A-UPWM pulse edges as multiples of the PWM fundamental period $T_{p}$. Again, the above time-domain minimization of the NPWM and AUPWM pulse edges positions is theoretically limited within the range [- LST /2, LST /2] due to the $N$-bit quantization of the PWM time domain.

\section{3. "Jither" realization}

Following the set of (18), the exact "jither" values in the amplitude domain can be calculated, provided that the input sample values $s\left(t_{\text {lead }, k}\right)$ and $s\left(t_{\text {trail }, k}\right)$ are already known. The same stands in the time-domain "jither" calculation, where the sampling instances $t_{\text {lead, } k}$ and $t_{\text {trail }, k}$ were assumed to be known in (19). However, this assumption is impractical in the case of digital PWM conversion, as it requires the presence of the analog version of the input signal.

In order to overcome the above problem, a novel algorithm was developed and is described in this paragraph for providing a very close estimation of the above-unknown values. It should be noted that, although the following analysis of the proposed algorithm focuses on time-domain "jither," it could be similarly described in the case of amplitude-domain “jither" as well.

Using the set of (19) and taking into account (4a), the proposed algorithm iteratively provides an estimation of the $k$ th PWM pulse leading edge time instance as

$$
m_{\mathrm{lead}, k}^{i+1}=\left[2 k+1-\frac{s\left(m_{\mathrm{lead}, k}^{i} T_{p}\right)}{S_{\max }}\right]\left(2^{N}-1\right),
$$

where $i$ is an integer that denotes the iteration index for the current "jither" value estimation. Obviously, for $i=0$, the value $s\left(m_{\text {lead }, k}^{0} T_{p}\right)$ equals to $s\left(k T_{s}\right)$ and the resulting $m_{\text {lead }, k}^{1} T_{p}$ value represents the leading edge instance of the legacy AUPWM described in Section 2. The above iterative process is repeated until the following condition is validated:

$$
\left|m_{\text {lead }, k}^{i+1}-m_{\text {lead }, k}^{i}\right| \leq D_{\tau}
$$

where $D_{\tau}$ is a positive nonzero integer that defines the accuracy (i.e., the degree of approximation of the AUPWM and NPWM) as multiple of the LST, that is $\left[-D_{\tau}(\mathrm{LST} / 2), D_{\tau}(\mathrm{LST} / 2)\right]$. Clearly, when $D_{\tau}=1$, the maximum theoretic approximation accuracy is achieved imposed by (19), due to the time-domain quantization of the AUPWM pulse edges within the range [-LST/2, LST/2]. As it will be shown later, the highest this approximation accuracy is, the largest number of iterations is performed and the corresponding computational load required for realizing the A-UPWM and NPWM approximation is increased.

In (21) the input signal value $s\left(m_{\text {lead }, k}^{i} T_{p}\right)$ must be also calculated. For this reason, the original digital audio input is oversampled prior to PWM conversion and the "jithering" process, typically by a factor $\times R_{v}$. As it will be shown later, this oversampling process does not affect the final PWM rate $f_{p}$, hence it is termed here as "virtual" oversampling. After virtual oversampling, in each input signal sampling period $T_{s}$, a total number of $R_{v}$ input signal values are available, denoted as $s\left(k T_{s}\right), s\left(k T_{s}+T_{s, R}\right), \ldots, s\left(k T_{s}+r T_{s, R}\right), \ldots, s\left(k T_{s}+\right.$ $\left.\left(R_{v}-1\right) T_{s, R}\right)$ where $T_{s, R}=T_{s} / R_{v}$. During the $i$ th iteration step of $(21)$, the samples $s\left(k T_{s}+r_{i} T_{s, R}\right)$ and $s\left(k T_{s}+\left(r_{i}+1\right) T_{s, R}\right)$ are selected which satisfy the equation

$$
k T_{s}+r_{i} T_{s, R} \leq m_{\text {lead }, k}^{i} T_{p} \leq k T_{s}+\left(r_{i}+1\right) T_{s, R}
$$

and these samples are employed for calculating the desired signal value $s\left(m_{\mathrm{lead}, k}^{i} T_{p}\right)$ using linear approximation, that is,

$$
\begin{aligned}
s\left(m_{\text {lead }, k}^{i} T_{p}\right)= & s\left[k T_{s}+r_{i} T_{s, R}\right] \\
& +\frac{s\left[k T_{s}+\left(r_{i}+1\right) T_{s, R}\right]-s\left[k T_{s}+r_{i} T_{s, R}\right]}{T_{s, R}} \\
& \times\left[m_{\text {lead }, k}^{i} T_{p}-\left(k T_{s}+r_{i} T_{s, R}\right)\right] .
\end{aligned}
$$




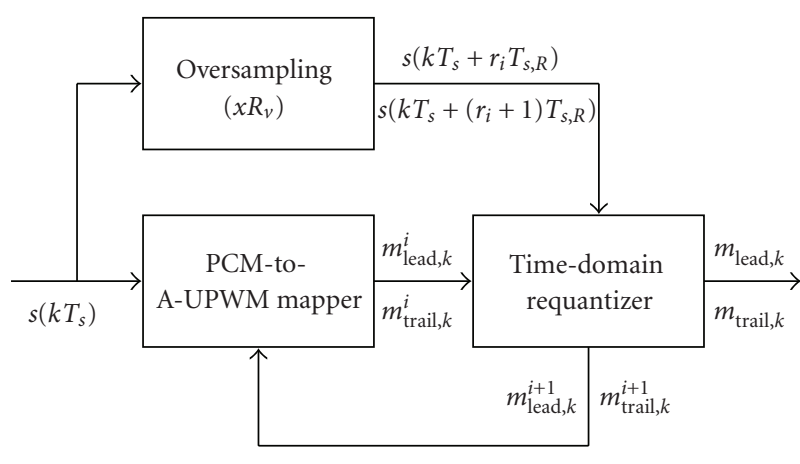

FIGURE 4: Block diagram of the proposed "jither" implementation algorithm in the time domain.

The same calculations' sequence is followed in the case of trailing edge time instance using the equation

$$
m_{\text {trail }, k}^{i+1}=\left[2 k+1+\frac{s\left(m_{\text {trail }, k}^{i} T_{p}\right)}{S_{\max }}\right]\left(2^{N}-1\right)
$$

until

$$
\left|m_{\text {trail }, k}^{i+1}-m_{\text {trail }, k}^{i}\right| \leq D_{\tau} .
$$

The above "jither" values estimation procedure is summarized in Figure 4. The iteration path between the PCM-toA-UPWM mapper and the time-domain requantizer that realizes (21) and (25) is followed until the conditions described by (22) and (26) are reached. In this case, the algorithm outputs the values $m_{\text {lead, } k}^{\prime}$ and $m_{\text {trail }, k}^{\prime}$ which define the "jithered" leading and trailing edges of each PWM pulse, respectively.

It should be also noted that, in the above analysis, the PWM pulse repetition rate equals to $f_{s}$ (the digital input signal sampling frequency). Hence, although virtual oversampling is employed, the final PWM clock rate is not proportionally increased. Moreover, due to the time-domain requantization stage which appeared in Figure 4, the optional requantizer module which appeared in Figure 3 is not necessary, as the appropriate selection of the $D_{\tau}$ parameter value results into the direct requantization of the input signal into the time domain. For example, assuming that the original bit resolution of signal $s\left(k T_{s}\right)$ equals to $N$, a value $D_{\tau}=2^{N^{\prime}}$ would result into requantization to $\left(N-N^{\prime}\right)$ bits, while for $D_{\tau}=1\left(N^{\prime}=0\right)$, no requantization is performed.

\section{RESULTS AND IMPLEMENTATION}

\subsection{Harmonic distortion suppression}

Figure 5 shows the 1-bit PWM spectrum in the case of a full-scale $(0 \mathrm{~dB}$ relative full scale, dB-FS) $5 \mathrm{kHz}$ sinewave signal, originally sampled at $f_{s}=44.1 \mathrm{kHz}$ and quantized using 16 bit. When $\times 2$ oversampling is applied on the input data, the UPWM spectrum contains the well-known even and odd numbered harmonics. No intermodulation products are present due to the $\times 2$ oversampling. Moreover, in this case, as no requantization is applied, the noise floor level

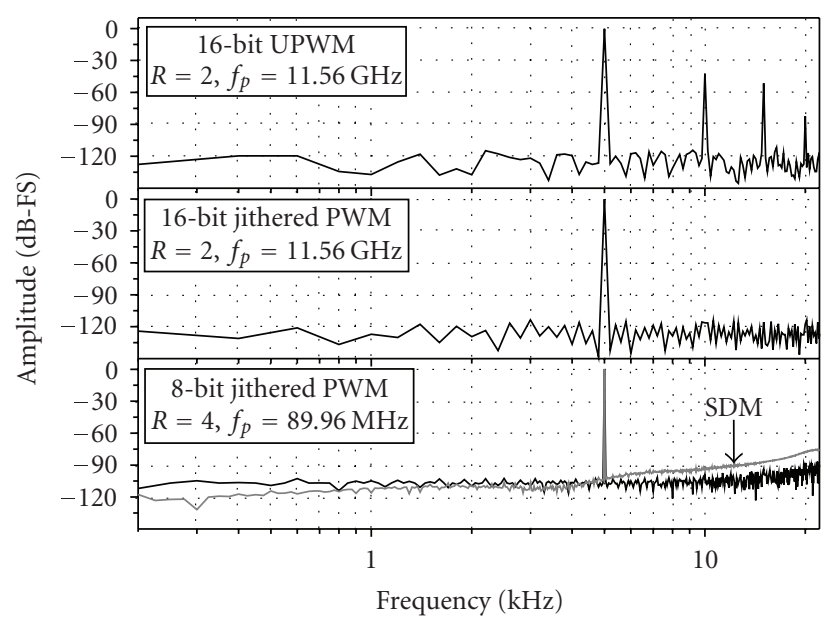

FIGURE 5: "Jither" effect on the final PWM spectrum in the case of $5 \mathrm{kHz}, 0 \mathrm{~dB}-\mathrm{FS}$ sinewave signal $\left(f_{s}=44.1 \mathrm{kHz}\right)$.

is equivalent to a 16-bit PCM signal and the final PWM clock rate equals to $f_{p}=11.56 \mathrm{GHz}$. Under the same clock rates, when "jithering" is applied (using $R_{v}=32$ for optimized performance as described in the following section), all harmonic intermodulation products are eliminated.

Although the above example clearly demonstrates the efficiency of the proposed "jithering" technique, the excessive final PWM clock rate value debars any practical realization of such a system. However, if time-domain requantization to $N^{\prime}=8$ bit (i.e., $D_{\tau}=2^{8}$ ) is assumed, the PWM clock rate is significantly reduced in the practically feasible range of 89.96 MHz, while the derived 1-bit PWM spectrum remains free of harmonic distortion. It should be also noted that in this case, $\times 4$ oversampling and 3 rd order noise shaping were also applied in order to reduce the average level of the 8-bit quantization noise within the lower audible frequency range.

In the same figure, the spectra of a 3rd order SDM modulator 1-bit output in the case of the same full-scale $5 \mathrm{kHz}$ sinewave signal are also shown. In this case, $\times 64$ oversampling was applied, resulting into a final SD clock rate equal to $2.8224 \mathrm{MHz}$. The noise floor level within the audible frequency band is almost identical for both 1-bit coding techniques. Moreover, although the SDM pulse switching rate is much lower than the $89.96 \mathrm{MHz}$ PWM clock rate, the actual PWM switching frequency equals to $4 \times 44.1=176.4 \mathrm{kHz}$. Hence, as previously discussed, the power dissipation for the PWM coding case will be significantly lower than for SDM coding.

In the following paragraphs an 8-bit time-domain requantization for the PWM coding is considered.

\section{2. "Jithering" parameter optimization}

The above results were obtained for a virtual oversampling factor equal to $R_{v}=32$. This value was found to be optimal after a sequence of tests that assessed the effect of the virtual oversampling factor on the amplitude of the harmonics of the input signal during PCM-to-PWM conversion. It should 


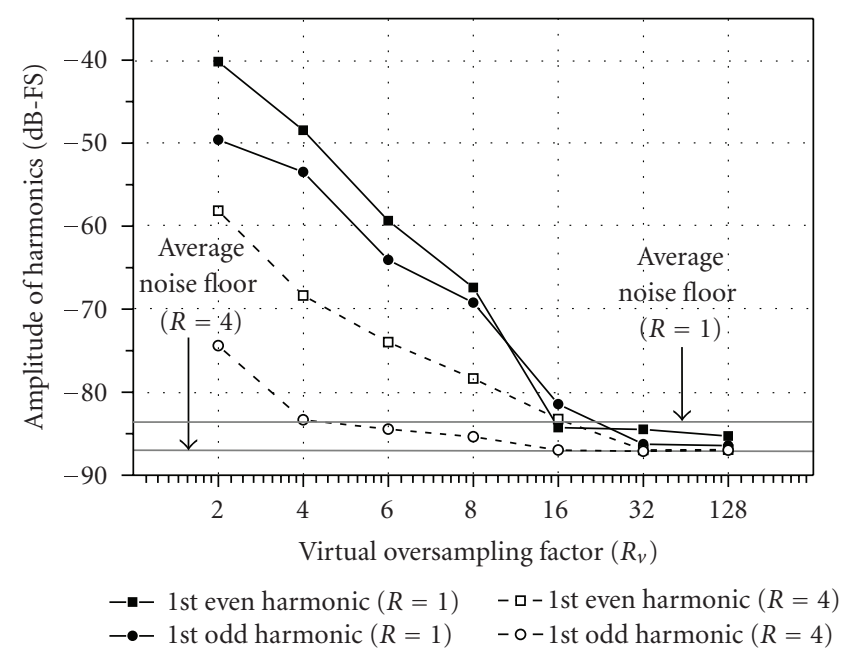

FIGURE 6: Variation of the "jithered" PWM harmonic amplitude with the virtual oversampling factor $R_{v}\left(D_{\tau}=1\right)$.

be noted that this amplitude is directly related to the approximation accuracy of the UPWM and NPWM coding schemes (the lowest the harmonic amplitude is, the highest approximation accuracy is achieved). In Figure 6 a typical example of the results obtained from these tests for a $5 \mathrm{kHz}$, full scale sinewave input is illustrated, showing the variation of the first even and odd harmonics amplitudes as a function of $R_{v}$, for $R=1$ and $R=4$. Clearly, in both cases the amplitude of the harmonics is suppressed to the corresponding average noise floor level for $R_{v}=32$ or more. This observation was verified in all tests performed for a variety of input sinewave frequencies. Hence, given that larger values of virtual oversampling require higher amounts of memory for storing the virtually oversampled samples, $R_{v}=32$ is considered to be the optimal choice.

When considering a specific $R_{v}$ parameter value, the approximation accuracy of the "jithered" PWM and NPWM coding schemes expressed in terms of the presented harmonic distortions is controlled and defined by the $D_{\tau}$ parameter. As discussed in Section 4, this parameter controls the repetitive execution of the "jither" values estimation using the condition described by (22) in the time domain. Figure 7 illustrates the effect of $D_{\tau}$ on the amplitude of the harmonics in both cases of $R=1$ and $R=4$ for a $5 \mathrm{kHz}$, full-scale sinewave signal. $R_{v}$ was equal to 32 , as analyzed previously, while 16 to 8 bit quantization was employed during PCM-toPWM conversion. Clearly, a small value of $D_{\tau}$ (i.e., $D_{\tau}=1$ ) results into harmonic distortions in the range of the mean quantization noise level, while larger values increase the amplitude of these distortions, due to the larger time-domain difference of the "jithered" PWM and NPWM modulations.

\subsection{Real-time implementation issues}

The proposed "jithering" PWM-distortion suppression scheme is based on an iterative signal estimation process. In any real-time implementation (e.g., on a digital signal pro-

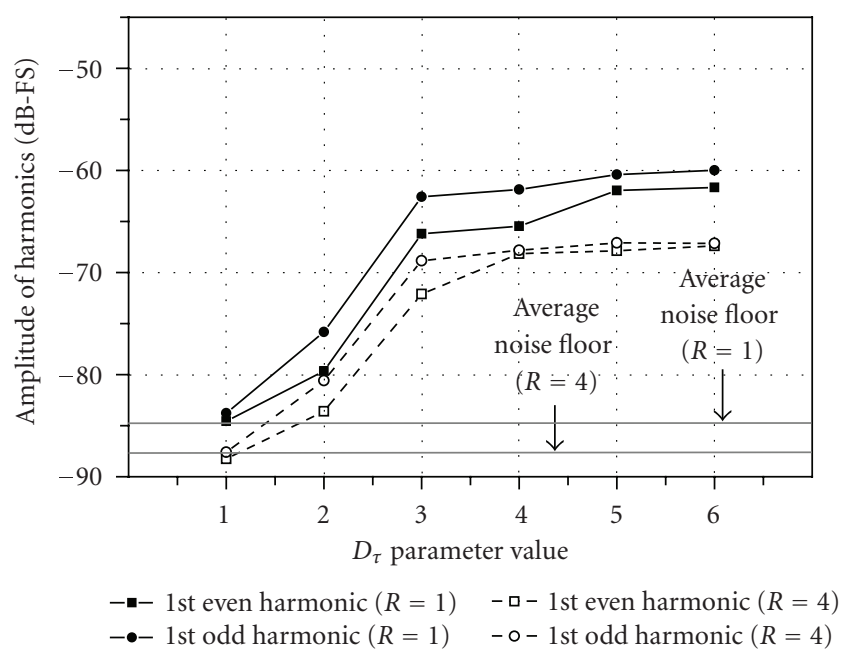

FIGURE 7: Variation of the "jithered" PWM harmonic amplitude with the $D_{\tau}$ parameter $\left(R_{v}=32\right)$.

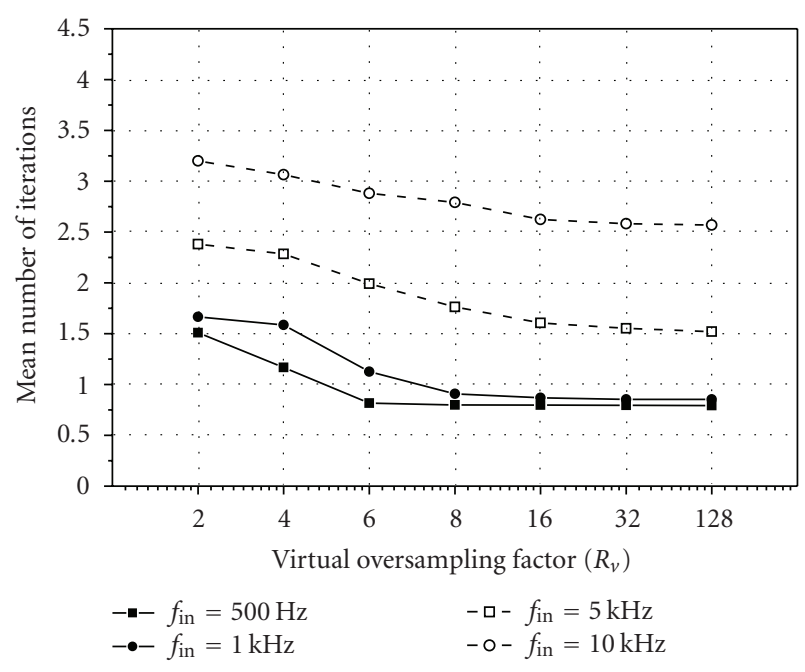

FIGURE 8: Mean iterations per PCM sampling period versus virtual oversampling factor $R_{v}\left(D_{\tau}=1, R=1\right)$.

cessor platform), the total number of iterations performed for the estimation of the leading and trailing edges "jither" values for each PCM sample must be executed before the expiration of the sampling period length. Hence, the determination of the number of the iterations necessary for producing the appropriate "jither" values is a very critical task.

As it is shown in Figures 8 and 9, this number of iterations depends on the $R_{v}$ and $D_{\tau}$ parameter values, as well as the input sinewave frequency. More specifically, as illustrated in Figure 8, the measured mean number of iterations of a variable frequency, full-scale sinewave signal decreases with the virtual oversampling factor due to the faster UPWM and NPWM approximation that can be achieved when more virtual samples are present, while it increases with the input sinewave frequency, due to the steeper signal transitions 


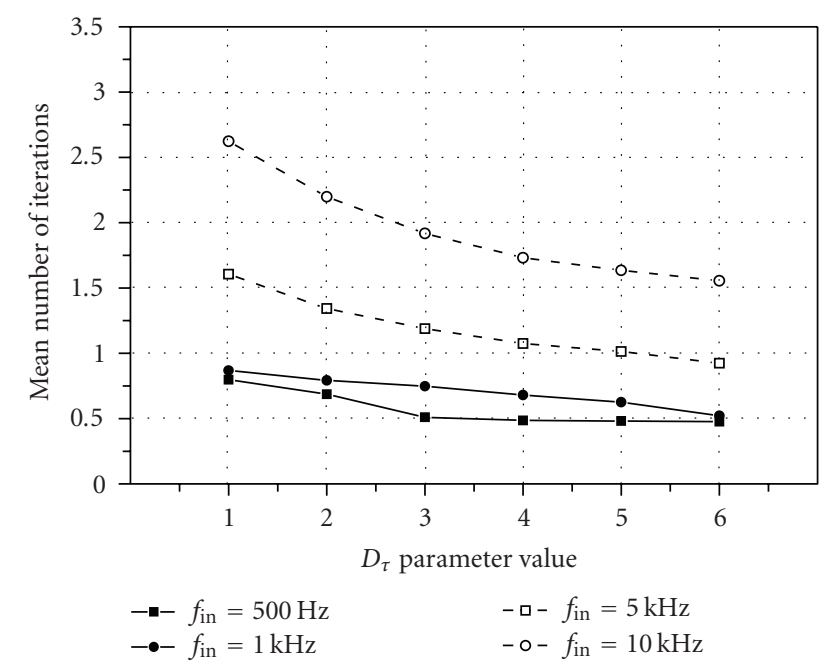

FIgURE 9: Mean iterations per PCM sampling period versus $D_{\tau}$ parameter $\left(R_{v}=32, R=1\right)$.

TABLE 1: Maximum number of iterations (for $R=4, R_{v}=32$, and $\left.D_{\tau}=1\right)$.

\begin{tabular}{l|c|c|c}
\hline Waveform type & $I_{L}$ & $I_{T}$ & $I_{L}+I_{T}$ \\
\hline $20 \mathrm{kHz}$ full-scale sinewave & 5 & 5 & 10 \\
Typical audio material & 6 & 6 & 12 \\
\hline
\end{tabular}

occurring for the increased sinewave frequency. Moreover, from the same figure it is obvious that the value $R_{v}=32$ (found to be optimal in the previous paragraph in terms of harmonic distortion suppression) is also optimal in terms of the number of iterations.

The same trends are observed when the mean number of iterations for both leading and trailing edges is measured as a function of the $D_{\tau}$ parameter. As it is shown in Figure 9, low $D_{\tau}$ values (i.e., high approximation accuracy) results into higher mean iterations number. The same is observed when the input sinewave frequency is increased.

The above results were based on the mean iterations' values in order to assess the dependency of iterations on the "jithering" algorithm parameters. However, in order to evaluate the real-time capabilities of the proposed algorithm, the maximum number of iterations observed among all PCM sampling periods must be considered, as it represents the worst case scenario in terms of the induced computational load. Let $I_{L}$ and $I_{T}$ be the maximum number of the iterations required for producing the final "jithered" leading and trailing edge values during the PCM-to-PWM conversion of an audio signal. Table 1 shows the measured $I_{L}$ and $I_{T}$ values in the case of a $20 \mathrm{kHz}$ full scale sinewave signal, as well as for a typical PCM audio waveform. As discussed in the previous section, $R_{v}$ was set equal to 32 , while $D_{\tau}=1$.

The above $I_{L}$ and $I_{T}$ values can be used for determining the computational requirements of a possible real-time implementation. As a fixed number of multiplications and additions is required for each iteration step (to implement (24)), the resulting computational load is simply propor- tional to the number of iterations performed for every input PCM sample. In the worst case, taking into account that the above maximum number of iterations must be accomplished within a single PCM sampling period and assuming that $T_{i}$ (in seconds) is the time required for a single iteration, then the condition for realizing the "jithering" process in real-time can be expressed as

$$
T_{s}=R\left[\left(I_{L}+I_{T}\right) T_{i}+T_{c}\right],
$$

where $T_{c}$ (in seconds) denotes a constant delay imposed by signal processing applied within each PCM sampling period (such as virtual oversampling and quantization of the oversampled data). It is also obvious that if $\times R$ oversampling is also applied, then the above condition is further deteriorated, as the PCM sampling period is reduced by $R$.

Both $T_{i}$ and $T_{c}$ values depend on the targeted hardware platform. Hence, the decision of developing the "jithering" PWM distortion suppression strategy on a specific digital signal processor should be based on (27) and the maximum values of $I_{L}$ and $I_{T}$ provided in Table 1 .

\subsection{Overall "jither" method performance}

The spectral results obtained previously as case studies, were verified by many additional tests, using as input both sinewave test signals and typical audio waveforms. In all cases, the performance achieved by using "jither" in the PCM amplitude domain was identical to that by using "jither" in the PWM time domain and in all cases a complete suppression of PWM distortions was achieved. Here, typical cumulative results are shown for the worst case input signals [22], by considering the performance of the proposed method using a full scale sinewave signal of varying frequency. Figure 10 shows the measured amplitude of the first even and odd harmonic for the cases of UPWM and "jithered" PWM conversion, as functions of the input sinewave frequency. Clearly, the "jithering" process reduces the amplitude of these distortion artifacts to the PCM noise floor level.

Figure 11 shows the total harmonic distortion (THD + noise) expressed in $\mathrm{dB}$, measured for the cases of PCM, UPWM, and the "jithered" PWM, as function of the input frequency for a 16-bit full scale input sinewave signal with $\times 4$ initial oversampling. Clearly, the use of the proposed method decreases the THD + noise to the level of the $\times 4$ oversampled source PCM signal, rendering it constant and input signal independent within the audio frequency band.

\section{CONCLUSIONS}

In this paper, it was shown that UPWM can meet highfidelity audio performance targets, after introduction of suitable signal conditioning based on the minimization of the differences between the A-UPWM and NPWM conversion (with the additional use of mild oversampling to remove the NPWM-induced nonharmonic artifacts outside the audio bandwidth). A novel methodology was introduced based on the detailed description of all the above signals. It was shown that the minimization of UPWM harmonic distortion 


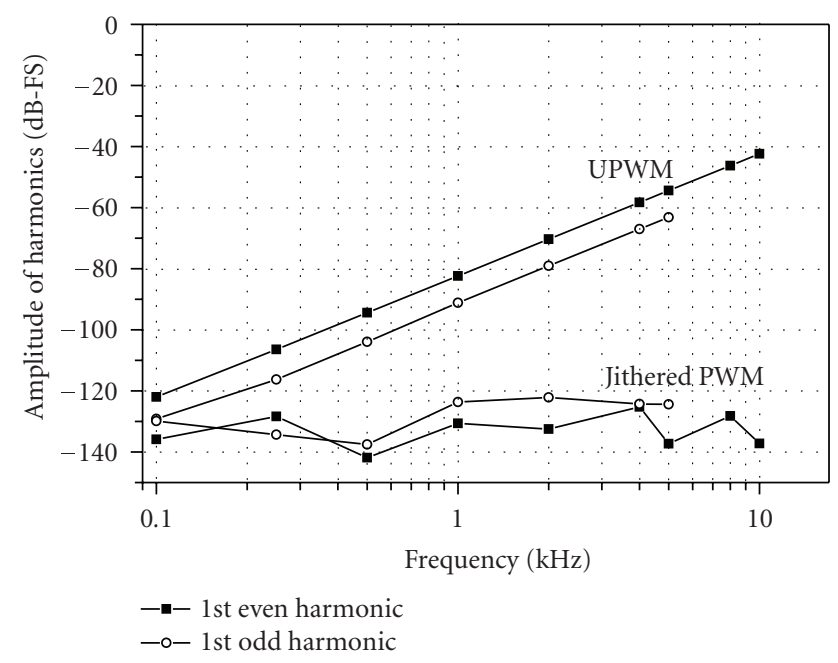

FIGURE 10: Measured 1st and 2nd harmonic amplitude for different input frequencies of $0 \mathrm{~dB}$-FS sinewave $\left(N^{\prime}=16\right.$ bit, $R=4, R_{v}=32$, and $\left.D_{\tau}=1\right)$.

artifacts can be achieved by two alternative but equivalent strategies, using "jither" (i.e., a novel 1-bit jitter signal having dither properties), either in the PCM multibit audio domain, or directly in the PWM stream.

It was shown that the above approach presents a number of theoretical and practical advantages compared to previously proposed methods and implementations. Specifically the following.

(a) It introduces an analytical description of all forms of PWM conversion, which allows the exact estimation of the PCM-to-PWM mapping errors and distortions. This description is not restricted to ideal harmonic input signals but it is applicable to all practical audio signals.

(b) A novel method ("jithering") for controlled jittering artifacts of the pulses of 1-bit digital PWM signals has been introduced for minimizing the distortions generated by mapping from multibit PCM signals.

(c) The proposed approach achieves adequate suppression of the UPWM-induced harmonic artifacts, rendering UPWM an audio-transparent process and equivalent to PCM as well as SDM coding, without requiring excessive oversampling and related prohibitively high clock rates. As it was shown, the reduction achieved in the amplitude of the harmonic UPWM distortions was up to $80 \mathrm{~dB}$ for the worst case of input signals examined. Moreover, compared to the SDM 1-bit modulation, the proposed method incorporates a significantly lower switching frequency, a parameter that directly affects the power dissipation and the resulting amplification efficiency in all-digital audio amplifier implementations, at the expense of increased implementation complexity.

(d) This algorithmic optimization approach allows exact prediction for any choice of system parameters (e.g., clock rate, PCM quantization accuracy, oversampling) in order to meet desired performance targets. A practical realization of a digital audio UPWM system could be achieved for clock rates in the region of $90 \mathrm{MHz}$.

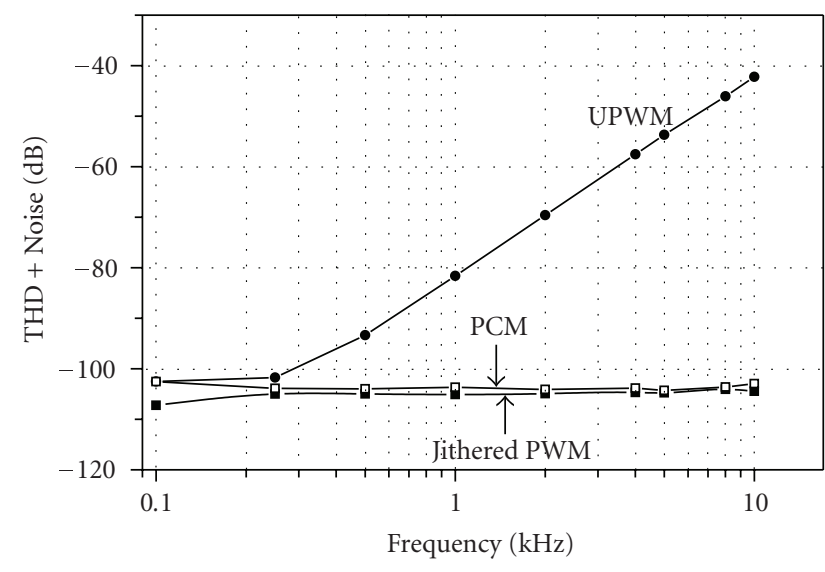

FIGURE 11: Measured THD + noise for different input frequencies of $0 \mathrm{~dB}$-FS sinewaves $\left(N^{\prime}=16 \mathrm{bit}, R=4, R_{v}=32\right.$, and $\left.D_{\tau}=1\right)$.

Various issues concerning the real-time implementation of the proposed approach were also described, focusing on parameters optimization and low implementation complexity targeted to current DSP hardware technology.

Possible future extension of this work will be also considered for the case of 1-bit digital inputs to the "jithered" PWM coder (e.g., SDM/DSD) and their direct and transparent conversion to distortion-free PWM, in order to take advantage of the superior PWM power performance and realize universal all-digital audio amplification systems.

\section{APPENDIX}

The following discussion aims to determine the input signal conditions (if any) that render UPWM 1-bit modulation equivalent to the multibit PCM coding, without employing any distortion suppression technique for reducing the PWMinduced distortions.

In (8) if we assume that $L_{1, k}=a_{q}\left(k T_{s}\right)\left(2^{N}-1\right)$ and $L_{2, k}=$ $a_{q}\left(k T_{s}+T_{s} / 2\right)\left(2^{N}-1\right)$, then the analytic time-domain representation of the 1-bit width modulated asymmetric pulses can be expressed as

$$
\begin{aligned}
\operatorname{PWM}(m)=A \sum_{k=0}^{d-1}\{ & u\left[m-\left((2 k+1)\left(2^{N}-1\right)-L_{1, k}\right)\right] \\
& \left.-u\left[m-\left((2 k+1)\left(2^{N}-1\right)+L_{2, k}\right)\right]\right\},
\end{aligned}
$$

where $d$ is the total number of the digital input samples converted to PWM pulses. Without loss of generality and under the assumptions made in [18], the discrete time function $\operatorname{PWM}(m)$ can be expressed in the form of Fourier series as

$$
\begin{aligned}
& \operatorname{PWM}(m)= \\
& \frac{\alpha_{0}}{2}+\sum_{\lambda=1}^{\infty}\left\{\alpha_{\lambda} \cos \left(\frac{2 \pi \lambda m}{2\left(2^{N}-1\right) d}\right)+b_{\lambda} \sin \left(\frac{2 \pi \lambda m}{2\left(2^{N}-1\right) d}\right)\right\},
\end{aligned}
$$


where $\alpha_{\lambda}$ and $b_{\lambda}$ are the Fourier series coefficients defined as

$\alpha_{\lambda}=\frac{2 A}{\pi \lambda} \sum_{k=0}^{d-1} \cos \left(\frac{\pi \lambda}{d}\left(2 k+1+\frac{L_{2, k}-L_{1, k}}{2\left(2^{N}-1\right)}\right)\right) \sin \left(\frac{\pi \lambda}{d} \frac{L_{2, k}+L_{1, k}}{2\left(2^{N}-1\right)}\right)$

$b_{\lambda}=\frac{2 A}{\pi \lambda} \sum_{k=0}^{d-1} \cos \left(\frac{\pi \lambda}{d}\left(2 k+1+\frac{L_{2, k}-L_{1, k}}{2\left(2^{N}-1\right)}\right)\right) \sin \left(\frac{\pi \lambda}{d} \frac{L_{2, k}+L_{1, k}}{2\left(2^{N}-1\right)}\right)$,

$\alpha_{0}=\frac{2 A}{d} \sum_{k=0}^{d-1}\left[\frac{L_{2, k}+L_{1, k}}{2\left(2^{N}-1\right)}\right]$.

The above equations can be expressed in exponential form as

$$
c_{\lambda}=\left\{\begin{array}{rlrl}
\frac{d A}{\pi \lambda} \sum_{k=0}^{d-1} & \sin \left(\frac{\pi \lambda}{d} \frac{L_{2, k}+L_{1, k}}{2\left(2^{N}-1\right)}\right) & \\
& \times e^{-j(\pi \lambda / d)\left(2 k+1+\left(L_{2, k}-L_{1, k}\right) / 2\left(2^{N}-1\right)\right)}, & \lambda \neq 0, \\
A \sum_{k=0}^{d-1} \frac{L_{2, k}+L_{1, k}}{2\left(2^{N}-1\right)}, & \lambda=0,
\end{array}\right.
$$

which describes the spectrum of all types of double-sided PWM. More specifically, if $L_{2, k}=L_{1, k}=L_{k}=a_{q}\left(k T_{s}\right)\left(2^{N}-\right.$ 1), (A.4) describes the UPWM spectrum generated from the conversion of the PCM signal $s_{q}\left(k T_{s}\right)$, while the spectral representation of the NPWM modulation is obtained for $L_{1, k}=$ $\left(s\left(t_{\text {lead }, k}\right) / S_{\max }\right)\left(2^{N}-1\right)$ and $L_{2, k}=\left(s\left(t_{\text {trail }, k}\right) / S_{\max }\right)\left(2^{N}-1\right)$.

Using the same methodology it can be also found [25] that the spectrum of the PCM signal corresponding to the $\mathrm{d}$ samples $s_{q}\left(k T_{s}\right)$ is given by

$$
c_{\lambda}^{\mathrm{PCM}}= \begin{cases}\frac{d}{\pi \lambda} \sum_{k=0}^{d-1} s_{q}\left(k T_{s}\right) \sin \left(\frac{\pi \lambda}{d}\right) e^{-j(\pi \lambda / d)(2 k+1)}, & \lambda \neq 0, \\ \sum_{k=0}^{d-1} s_{q}\left(k T_{s}\right), & \lambda=0 .\end{cases}
$$

Hence, the spectral representation of the difference between the PCM coding and the UPWM conversion can be defined as

$$
\begin{aligned}
E_{\lambda}= & c_{\lambda}^{\mathrm{UPWM}}-c_{\lambda}^{\mathrm{PCM}} \\
= & \frac{d}{\pi \lambda} \sum_{k=0}^{d-1}\left[A \sin \left(\frac{\pi \lambda}{d} \frac{s_{q}\left(k T_{s}\right)}{S_{\max }}\right)-s_{q}\left(k T_{s}\right) \sin \left(\frac{\pi \lambda}{d}\right)\right] \\
& \times e^{-(\pi \lambda / d) j(2 k+1)}, \quad \lambda \neq 0 .
\end{aligned}
$$

Assuming now that $S_{\max }=A$ and given that

$$
\sin x=x-\frac{x^{3}}{3 !}+\frac{x^{5}}{5 !}-\frac{x^{7}}{7 !}+\cdots, \quad-\infty<x<\infty,
$$

(A.6) results into

$$
\begin{aligned}
& E_{\lambda}=\frac{d A}{\pi \lambda} * \\
& \sum_{k=0}^{d-1}\left\{a_{q}\left(k T_{s}\right)\left[\sum_{l=1}^{\infty}(-1)^{l} \frac{a_{q}^{2 l}\left(k T_{s}\right)-1}{(2 l+1) !}\left(\frac{\pi \lambda}{d}\right)^{2 l+1}\right] e^{-j(\pi \lambda / d)(2 k+1)}\right\} .
\end{aligned}
$$

Clearly, the above spectral difference equals to zero for all $\lambda$ when $a_{q}\left(k T_{s}\right)=1$, that is $s_{q}\left(k T_{s}\right)=A$. In this case, both PCM and UPWM waveforms have exactly the same spectral characteristics. Hence, PCM coding and UPWM 1-bit modulation are equivalent only is the case of a full-scale DC digital input signal.

\section{REFERENCES}

[1] A. Nishio, G. Ichimura, Y. Inazawa, N. Horikawa, and T. Suzuki, "Direct stream digital audio system," in Proceedings of the 100th Convention of Audio Engineering Society (AES '96), Copenhagen, Denmark, May 1996, preprint 4163.

[2] J. Verbakel, L. van de Kerkhof, M. Maeda, and Y. Inazawa, "Super audio CD format," in Proceedings of the 104th Convention of Audio Engineering Society (AES '98), Amsterdam, The Netherlands, May 1998, preprint 4705.

[3] J. M. Goldberg and M. B. Sandler, "Pseudo-natural pulse width modulation for high accuracy digital-to-analogue conversion," Electronics Letters, vol. 27, no. 16, pp. 1491-1492, 1991.

[4] K. Nielsen, "A review and comparison of pulse width modulation (PWM) methods for analog and digital input switching power amplifiers," in Proceedings of the 102nd Convention of Audio Engineering Society (AES '97), Munich, Germany, March 1997, preprint 4446.

[5] K. Nielsen, "Linearity and efficiency performance of switching audio power amplifier output stages-a fundamental analysis," in Proceedings of the 105th Convention of Audio Engineering Society (AES '98), San Francisco, Calif, USA, September 1998 , preprint 4838.

[6] M. J. Hawksford, "Modulation and system techniques in PWM and SDM switching amplifiers," Journal of the Audio Engineering Society, vol. 54, no. 3, pp. 107-139, 2006.

[7] R. Esslinger, G. Gruhler, and R. W. Stewart, "Digital power amplification based on pulse-width modulation and sigmadelta loops. A comparison of current solutions," in Proceedings of the Institute of Radio Electronics, Czech and Slovak Radio Engineering Society (RADIOELEKTRONIKA '99), Brno, Czech Republic, April 1999.

[8] A. J. Magrath and M. B. Sandler, "Digital power amplification using sigma-delta modulation and bit flipping," Journal of the Audio Engineering Society, vol. 45, no. 6, pp. 476-487, 1997.

[9] M. J. Hawksford, "SDM versus PWM power digital-toanalogue converters (PDAC) in high-resolution digital audio applications," in Proceedings of the 118th Convention of Audio Engineering Society (AES '05), Barcelona, Spain, May 2005, preprint 6471.

[10] S. R. Bowes, "New sinusoidal pulsewidth-modulated invertor," IEE Proceedings, vol. 122, no. 11, pp. 1279-1285, 1975.

[11] M. J. Hawksford, "Linearization of multilevel, multiwidth digital PWM with applications in digital-to-analog conversion," Journal of the Audio Engineering Society, vol. 43, no. 10, pp. 787-798, 1995. 
[12] J.-W. Jung and M. J. Hawksford, "An oversampled digital PWM linearization technique for digital-to-analog conversion," IEEE Transactions on Circuits and Systems, vol. 51, no. 9, pp. 1781-1789, 2004.

[13] K. Nielsen, "High-fidelity PWM-based amplifier concept for active loudspeaker systems with very low energy consumption," Journal of the Audio Engineering Society, vol. 45, no. 7-8, pp. 554-570, 1997.

[14] H. S. Black, Modulation Theory, Van Nostrand, Princeton, NJ, USA, 1953.

[15] P. H. Mellor, S. P. Leigh, and B. M. G. Cheetham, "Reduction of spectral distortion in class D amplifiers by an enhanced pulse width modulation sampling process," IEE Proceedings-Part G: Circuits, Devices and Systems, vol. 138, no. 4, pp. 441-448, 1991.

[16] S. R. Bowes and Y.-S. Lai, "Relationship between space-vector modulation and regular-sampled PWM," IEEE Transactions on Industrial Electronics, vol. 44, no. 5, pp. 670-679, 1997.

[17] S. R. Bowes and B. M. Bird, "Novel approach to the analysis and synthesis of modulation processes in power converters," IEE Proceedings, vol. 122, no. 5, pp. 507-513, 1975.

[18] A. Floros and J. Mourjopoulos, "Analytic derivation of audio PWM signals and spectra," Journal of the Audio Engineering Society, vol. 46, no. 7, pp. 621-633, 1998.

[19] S. Lipshitz, R. Wannamaker, and J. Vanderkooy, "Quantization and dither: a theoretical survey," Journal of the Audio Engineering Society, vol. 40, no. 5, pp. 355-375, 1992.

[20] R. M. Gray, "Quantization noise spectra," IEEE Transactions on Information Theory, vol. 36, no. 6, pp. 1220-1244, 1990.

[21] B. A. Blesser, "Digitization of audio: a comprehensive examination of theory, implementation, and current practice," Journal of the Audio Engineering Society, vol. 26, no. 10, pp. 739$771,1978$.

[22] A. Floros and J. Mourjopoulos, "A study of the distortions and audibility of PCM to PWM mapping," in Proceedings of the 104th Convention of Audio Engineering Society (AES '98), Amsterdam, The Netherlands, May 1998, preprint 4669.

[23] A. Floros, J. Mourjopoulos, and D. E. Tsoukalas, "Jither: the effects of jitter and dither for 1-bit audio PWM signals," in Proceedings of the 106th Convention of Audio Engineering Society (AES '99), Munich, Germany, May 1999, preprint 4656.

[24] P. Craven, "Toward the 24-bit DAC: novel noise-shaping topologies incorporating correction for the nonlinearity in a PWM output stage," Journal of the Audio Engineering Society, vol. 41, no. 5, pp. 291-313, 1993.

[25] A. Floros and J. Mourjopoulos, "On the nature of digital audio PWM distortions," in Proceedings of the 108th Convention of Audio Engineering Society (AES '00), Porte Maillot, Paris, France, February 2000, preprint 5123.

Andreas Floros was born in Drama, Greece in 1973. In 1996 he received his Engineering degree from the Department of Electrical and Computer Engineering, University of Patras, and in 2001 his Ph.D. degree from the same department. His research was mainly focused on digital audio signal processing and conversion techniques for all-digital power amplification methods. $\mathrm{He}$ was also involved in research in the area of

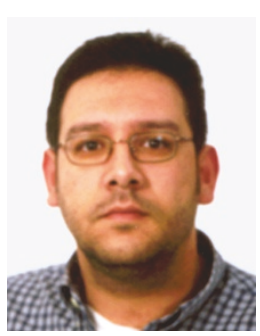
acoustics. In 2001, he joined ATMEL Multimedia and Communications, working in projects related with digital audio delivery over PANs and WLANs, quality-of-service, mesh networking, wireless
VoIP technologies, and lately with audio encoding and compression implementations in embedded processors. Since 2005, he is a visiting Assistant Professor at the Department of Audio Visual Arts, Ionian University. He is a Member of the Audio Engineering Society, the Hellenic Institute of Acoustics, and the Technical Chamber of Greece.

John Mourjopoulos was born in Drama, Greece, in 1954. In 1977, he received the B.S. degree in engineering from Coventry University in the United Kingdom and in 1979 the M.S. degree in acoustics from the Institute of Sound and Vibration Research (ISVR), University of Southampton. In 1984, he completed the Ph.D. degree at the same institute, working in the areas of digital signal processing and room acous-

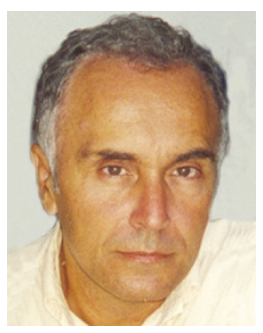
tics. He also worked at ISVR as a Researcher Fellow. Since 1986 he has been with the Wire Communications Laboratory, Electrical \& Computer Engineering Department, University of Patras, where he is currently an Associate Professor in electroacoustics and digital audio technology and Head of the Audio and Acoustics Technology Group. In 2000, during his sabbatical, he was a Visiting Professor at the Institute for Communication Acoustics at Ruhr-University Bochum, in Germany. He has organized many seminars and short courses in digital audio signal processing, has worked in the development of digital audio devices, and has authored and presented numerous papers in international journals and conferences. 